\title{
Chloroplast: The Emerging Battlefield in Plant-Microbe Interactions
}

\author{
Feng Yang, Kunqin Xiao, Hongyu Pan and Jinliang Liu* \\ College of Plant Sciences, Jilin University, Changchun, China
}

Higher plants and some algae convert the absorbed light into chemical energy through one of the most important organelles, chloroplast, for photosynthesis and store it in the form of organic compounds to supply their life activities. However, more and more studies have shown that the role of chloroplasts is more than a factory for photosynthesis. In the process of light conversion to chemical energy, any damage to

OPEN ACCESS

Edited by:

Lars Matthias Voll,

University of Marburg, Germany

Reviewed by:

Mario Serrano,

National Autonomous University

of Mexico, Mexico

Chanhong Kim,

Shanghai Institutes for Biological

Sciences (CAS), China

Vivek Dogra,

Institute of Himalayan Bioresource

Technology (CSIR), India

${ }^{*}$ Correspondence:

Jinliang Liu

jlliu@jlu.edu.cn

Specialty section:

This article was submitted to Plant Pathogen Interactions,

a section of the journal

Frontiers in Plant Science

Received: 04 December 2020

Accepted: 28 January 2021

Published: 03 March 2021

Citation:

Yang F, Xiao K, Pan H and Liu J (2021) Chloroplast: The Emerging Battlefield in Plant-Microbe

Interactions.

Front. Plant Sci. 12:637853.

doi: 10.3389/fpls.2021.637853 the components of chloroplast may affect the photosynthesis efficiency and promote the production of by-products, reactive oxygen species, that are mainly produced in the chloroplasts. Substantial evidence show that chloroplasts are also involved in the battle of plants and microbes. Chloroplasts are important in integrating a variety of external environmental stimuli and regulate plant immune responses by transmitting signals to the nucleus and other cell compartments through retrograde signaling pathways. Besides, chloroplasts can also regulate the biosynthesis and signal transduction of phytohormones, including salicylic acid and jasmonic acid, to affect the interaction between the plants and microbes. Since chloroplasts play such an important role in plant immunity, correspondingly, chloroplasts have become the target of pathogens. Different microbial pathogens target the chloroplast and affect its functions to promote their colonization in the host plants.

\section{Keywords: chloroplast immunity, phytohormone, light-harvesting complex, CAS, retrograde signaling pathway, effectors}

\section{INTRODUCTION}

Chloroplasts are considered to be organelles produced by endosymbiotic bacteria in plants that undergo photoautotrophy (Ding et al., 2019). Through coexistence and evolution in billions of years, the photosynthetic cyanobacteria, then chloroplasts, become indispensable parts of eukaryotic plant cells (Gray, 1989; Cavalier-Smith, 2002; Ziehe et al., 2017). During the coevolution process with host cells, most of the prokaryotic genomic contents of cyanobacteria are transferred to the host cell nucleus, resulting in approximately 100 genes in the current chloroplast genome (McFadden, 2001; Martin et al., 2002; Daniell et al., 2016). Most of the chloroplast-targeted proteins encoded in host nucleus contain a chloroplast transit peptide (cTP) at their N-terminal, which subsequently enters chloroplasts by interacting with the translocators located in the inner membrane (TIC) and outer membrane (TOC) of the chloroplast and could be cleaved off by a stromal processing peptidase (SPP) (Bruce, 2000; Jarvis and Soll, 2001; Day and Theg, 2018; 
Richardson et al., 2018). Some chloroplast-targeted proteins do not contain a cleavable N-terminal cTP, while they enter chloroplasts through some non-classical ways (Jarvis and Robinson, 2004; Richly and Leister, 2004; Beck, 2005; Bedard, 2005; Beale, 2011), and the examples include substrate-dependent import of pPORA proteins (Reinbothe et al., 2000). In addition, the Arabidopsis chloroplast heat shock proteins, AtcpHsp701 and AtcpHsp70-2, contain age-selective signals in transit peptides, which enable them to preferentially enter chloroplasts in mature stage (Teng et al., 2012).

Chloroplast is the organelle containing chlorophyll for photosynthesis and is the main source of reactive oxygen species (ROS), which can lead to a damage of the photosystem II (PSII) reaction center (Krieger-Liszkay et al., 2011). Overreduction of PSII and oxygen molecules by excessive illumination leads to the formation of ROS, including singlet oxygen $\left({ }^{1} \mathrm{O}_{2}\right)$ and superoxide anion radicals $\left(\mathrm{O}_{2}^{-}\right)$, and $\mathrm{O}_{2}^{-} \cdot$ dismutates to form free $\mathrm{H}_{2} \mathrm{O}_{2}$ that is then reduced to hydroxyl radical (HO.) (Pospísil et al., 2004). Overaccumulation of ROS could damage the plant growth and development. Meanwhile, the plant-defense-related hormones, salicylic acid (SA), and jasmonic acid (JA), for example, are synthesized in the chloroplasts.

Unlike animals, plants cannot move to escape the invasions from natural enemies, which consequently have evolved a complex multilayered immune system to protect themselves from microbial infection and insect feeding. Plasma membrane (PM)-localized pattern-recognition receptors (PRRs) consist of the first layer of immune system, which perceive the pathogenassociated molecular pattern (PAMP) and trigger immunity [PAMP-triggered immunity (PTI)]. On the other hand, certain specific effectors can be directly or indirectly recognized by plant intracellular nucleotide-binding leucine-rich repeat domain-containing receptor (NLR), second layer of the immune system, to trigger a robust immune response [effector-triggered immunity (ETI)] (Jones and Dangl, 2006; Zipfel, 2014; Liu C. et al., 2019). Once the presence of microbes has been perceived by plants, the signaling cascades are then activated, which in turn initiate a multilayered immune response, and the outputs of this combat, resistance or susceptibility, between plant and pathogens depends on several factors, in which chloroplasts play significant roles.

\section{FROM INVASION SIGNAL PERCEPTION TO CHILOROPLAST}

PAMP-triggered immunity and ETI immune responses vary largely in the magnitude and duration but result in similar downstream molecular events, such as mitogen-activated protein kinase (MAPK) activation, oxidative burst, ion influx, $\mathrm{Ca}^{2+}$ signaling, increased biosynthesis of plant defense hormones, and transcriptional reprogramming (Peng et al., 2018). After PAMP perception, several lines of evidence demonstrate that PTI response triggers $\mathrm{Ca}^{2+}$ influx at the plasma membrane (Ranf et al., 2011; Nomura et al., 2012). Although several proteins were suggested to be potential $\mathrm{Ca}^{2+}$ channels in plants, for example, ionotropic glutamate receptor-like channels (GLRs), cyclic nucleotide gated channels (CNGCs), and mechanosensitive MCA-like channels (MCAs), one of the most recent works indicate that CNGC2 and CNGC4 in Arabidopsis are essential for PAMP-induced $\mathrm{Ca}^{2+}$ signaling, which constitute a functional channel and is phosphorylated by receptor-like cytoplasmic kinase BIK1, a core PTI regulator, to increase the cytosolic calcium concentration (Tian et al., 2019). A similar finding in rice suggests that OsCNGC9 is phosphorylated by OsRLCK185 after PAMP treatment to activate the calcium channel (Wang et al., 2019), supporting the model that RLCK-mediated phosphorylation of CNGCs plays important roles in PTI. The identity of plasma membrane $\mathrm{Ca}^{2+}$ channels responsible for the $\mathrm{Ca}^{2+}$ influx in ETI is still unknown (Seybold et al., 2014), although many $\mathrm{Ca}^{2+}$-responsive proteins have been identified as critical regulators of plant immunity.

Calcium-sensing receptor (CAS) is a calcium-binding protein located on the thylakoid membrane in the chloroplast. Once PTI or ETI signal is relayed to the chloroplast, $\mathrm{Ca}^{2+}$ from the thylakoid lumen, which contains a high concentration of $\mathrm{Ca}^{2+}$, are transported to the stroma by CAS, resulting in a continuous high concentration of $\mathrm{Ca}^{2+}$ in the stroma, and the signal is then transduced from the chloroplast to the nucleus through the ${ }^{1} \mathrm{O}_{2}$-mediated retrograde signaling pathway (Kim and Apel, 2013), which regulates the defense responses through transcriptional reprogramming of defenserelated genes (Nomura et al., 2012). Another outstanding example is that the calcium protein kinase 16 (CPK16), localized on the PM, undergoes N-myristoylation in normal condition and relocalizes from the PM to chloroplasts upon flg22 or immune elicitors treatment to promote chloroplast-dependent defenses (Medina-Puche et al., 2020).

Both PTI and ETI responses activate MAPK pathway in a short and sustained manner. The sustained MPK3 and MPK6 activation, triggered by ETI response, could inhibit photosynthesis, decrease the $\mathrm{CO}_{2}$ fixation, increase the ROS accumulation and the programmed cell death (PCD), and increase the synthesis of defense-related secondary metabolites (Su et al., 2018). Once plants sense invasions, they can actively inhibit photosynthesis and thus allocate more energy to the immune responses (Nomura et al., 2012). On the other hand, pathogens could decrease the photosynthesis in plants by secreting metabolites and proteins (Rodríguez-Herva et al., 2012; Bhattacharyya et al., 2015; Schmid et al., 2016; Xu et al., 2019), which directly affect the accumulation of ROS (Rodríguez-Herva et al., 2012; Zhou et al., 2015; Xu et al., 2019). ROS are considered to play different roles in combating different pathogens, which promote the infection of necrotrophs while inhibiting biotrophs, since ROS is not only an important signaling molecule but also a toxic factor to cells that is often related to hypersensitive response (HR) and programmed cell death (PCD) (Govrin and Levine, 2000; Glazebrook, 2005).

Guanosine tetraphosphate [(p)ppGpp] is a regulator of chloroplast gene expression. Plants with excessive accumulation of (p)ppGpp show defects in chloroplast function, with upregulation of large amounts of chloroplast function-related genes, while plants with low (p)ppGpp levels show increased SA accumulation, premature expression of PR genes, and 
increased resistance to Turnip mosaic virus (TuMV) infection (Abdelkefi et al., 2018).

\section{IMMUNE RESPONSES INSIDE CHLOROPLAST}

The light-harvesting complex II (LHCII) surrounds PSII to absorb light and transmit to PSII (Sheng et al., 2018), in which the light is converted into chemical energy. Magnaporthe oryzae, a filamentous fungal pathogen, causes devastating rice blast disease on rice, and the light-induced phosphorylation of light-harvesting complex II protein (LHCB5) enhances broadspectrum resistance of rice to M. oryzae (Liu M. et al., 2019). The members of the LHCB family are important in photosynthesis and guard cell signaling in response to abscisic acid (ABA), while downregulation or disruption of LHCB leads to less sensitive to $\mathrm{ABA}$ and $\mathrm{ABA}-$-regulated stomatal movement in guard cells and partly destroys ROS homeostasis (Xu et al., 2012). ABA-mediated stomata closure can effectively prevent the invasion of microbes (Lim et al., 2015).

Intracellular ROS are mainly produced in the chloroplasts, which can act as signaling molecules to affect localized cell death (LCD) in non-host resistance. The non-host resistance 2 (NHR2) protein of tobacco and Arabidopsis was proposed to act as a new component of the chloroplast-signaling pathway to activate the callose deposition to the cell wall in response to bacterial pathogens and enhance non-host disease resistance (Singh et al., 2018). Tobacco overexpressing flavodoxin (Fld) accumulates less ROS in chloroplasts, which leads to the inhibition of LCD after inoculation of the non-adapted bacterium Xanthomonas campestris pv. vesicatoria (Pierella Karlusich et al., 2017). In addition, studies have shown that overexpression of plastidtargeted cyanobacterial flavodoxin (pfld) in tobacco results in significantly reduced accumulation of ROS in chloroplast, which in turn leads to the enhanced resistance to Botrytis cinerea, suggesting that ROS derived from chloroplast plays an important role in the resistance of plants to necrotrophic fungus (Rossi et al., 2017). The h-type thioredoxin TRXh3 in tobacco is specifically located in the chloroplast and maintains the reduced state of cells. Tobacco plants overexpressing TRXh3 show increased resistance to Tobacco mosaic virus (TMV) and Cucumber mosaic virus (CMV) (Sun et al., 2010).

\section{THE CHLOROPLAST: FACTORY FOR JA AND SA PRODUCTION}

The plant defense responses are based on a highly regulated and complex network of phytohormone signaling pathways, in which SA and JA are thought to be the backbone ( $\mathrm{Li}$ et al., 2019). Chloroplasts can regulate the production of SA and JA. The SA-mediated defense response restricts the spread of biotrophic and hemibiotrophic pathogens, while the JAmediated defense response mainly targets the necrotrophic pathogens. It is generally agreed that the SA- and JAmediated signaling pathways are mutually antagonistic. This notion is well supported by the case of a bacterial pathogen Pseudomonas syringae that secretes a phytotoxin coronatine (COR), mimicking the jasmonate-isoleuce (JA-Ile) to promote the degradation of the Jasmonate Zim domain (JAZ) protein and to activate the JA-induced defense, thus inhibiting the SA-mediated defense (Bender et al., 1998, 1999). Different from bacteria, in which isochorismate is directly converted to SA by an isochorismate pyruvate lyase, SA in plants is synthesized through the phenylalanine ammonia-lyase (PAL) and isochorismate synthase (ICS) pathways (Dempsey et al., 2011). Moreover, the accumulation of SA caused by pathogen invasion is mainly through ICS pathway. Chorismate, the product of the shikimate pathway, is catalyzed by ICS to form isochorismate in the chloroplast, which is then transported to cytoplasm by enhanced disease susceptibility 5 (EDS5) (Rekhter et al., 2019), and is then catalyzed by avrPphB Susceptible 3 (PBS3) to form IC-9-Glu, and is finally converted to SA spontaneously or catalyzed by enhanced pseudomonas susceptibility 1 (EPS1) (Torrens-Spence et al., 2019). Accumulation of SA was shown to be essential for systemic acquired resistance (SAR) (Gaffney et al., 1993). In the infected leaves, the syntheses of SA and pipecolic acid (Pip) are enhanced. Then, Pip and/or its derivative $\mathrm{N}$-pipecolic acid (NHP) moves through the phloem to the distal uninfected leaves (Chen et al., 2018), increase the stability of non-expresser of PR genes 1 (NPR1) protein, which then activates SA and Pip biosynthesis and SAR at low SA concentration (Kim et al., 2020; Sun et al., 2020). The precursor of JA biosynthesis (9S, 13S)-12-oxophytodienoic acid (OPDA) is synthesized in chloroplasts. The galactolipids on the chloroplast membranes are catalyzed by fatty acid desaturase (FAD) and phospholipase A1 (PLA1) to release $\alpha$-linolenic acid ( $\alpha$-LeA/18:3) (Wasternack and Song, 2017), which then synthesizes OPDA under the actions of 13-lipoxygenase (LOX), allene oxide synthase (AOS), and allene oxide cyclase (AOC); then, OPDA is transferred to the peroxisome to synthesize JA (Huang H. et al., 2017). In the process of $\mathrm{ABA}$ biosynthesis, the synthesis of carotenoid precursor and the formation and cleavage of xanthophyll all occur in plastids (Seo and Koshiba, 2002).

\section{STROMULES AND CHLOROPLAST MOVEMENT}

As a multicellular organism, communication between organelles is crucial for plant immune responses (Théry et al., 2009). Chloroplasts in tobacco epidermal cells are mainly located on the outer edge of the cell due to the extrusion of the huge central vacuole. Once the defense responses are activated, the chloroplasts quickly rearrange, cluster around the nucleus, and establish a connection with the nucleus through sending out dynamic tubular elongation, called stromules (Natesan et al., 2005). The stromule is a dynamic structure that depends on microtubules (MTs) and actin filaments (AFs). The top of the stromule interacts dynamically with MT and extends along the MT, which maintains their length by repeating the process of elongation and contraction. Although AFs are not 
required for the extension of stromule, they provide an anchor point. The stromules guide or push the chloroplast toward the nucleus by tightly binding the AFs around the nucleus, facilitating the relocalization of chloroplasts to nuclei during innate immunity (Kumar et al., 2018). The stromules connect the plastids and exchange the metabolites in the stroma (Hanson and Hines, 2018). N receptor interacting protein 1 (NRIP1), which localizes in the chloroplast normally, is recruited to the cytoplasm and nucleus during plant immunity (Caplan et al., 2008). Chloroplasts may transmit defense-promoting signals (such as NRIP1, $\mathrm{H}_{2} \mathrm{O}_{2}$ ) to the nucleus or other subcellular compartments through the stromules, and the formation of stromules precedes HR-PCD and pro-PCD signals (Serrano et al., 2016). Defense-related molecules $\mathrm{H}_{2} \mathrm{O}_{2}$ and $\mathrm{SA}$ can induce the formation of stromules. Since the pro-defense signals originate from the chloroplast, the increased surface area caused by the stromules may help their transfer to the cytoplasm and nucleus (Caplan et al., 2015). Ferredoxin2 is distributed in the chloroplasts clustered around the stromules and nucleus, indicating that it is likely to be transmitted from the chloroplast to the nucleus through the stromules (Wang et al., 2018). The accumulation of chloroplasts around the nucleus is a common response of plants upon activation of immune responses. In Nicotiana benthamiana, activation of PTI or ETI, transient expression of the replication-associated protein (Rep) from three different geminiviruses, or infection with RNA viruses or phytopathogenic bacteria can trigger this response (Kwok and Hanson, 2004; Caplan et al., 2008), which also occurs in noninfected cells. Therefore, it is very likely that a certain signal molecule produced by the chloroplast that is clustering around the nucleus can act as a system signal to transmit information, probably related to defense response, to eventually neighboring cells. Exogenous application of $\mathrm{H}_{2} \mathrm{O}_{2}$ is sufficient to induce the chloroplast perinuclear clustering, and the inhibition of intracellular ROS production reduces the number of clustered chloroplasts, indicating that ROS is necessary to induce the relocalization of chloroplasts. Therefore, this response is likely to be produced by sensing and accumulating ROS (Ding et al., 2019). In addition, the chloroplasts can also navigate toward the pathogen interface. During the infection of Phytophthora infestans, the $N$. benthamiana chloroplasts can navigate to the pathogen interface. Chloroplast unusual positioning 1 (CHUP1) is necessary for the repositioning of chloroplast by regulating the anchoring of chloroplast to the plant-derived extra-haustorial membrane (EHM). Chloroplasts can be recruited to the interface where the pathogen infection structure haustoria presents and establish contact with EHM by forming stromules. The plant cells may generate mechanical pressure through chloroplasts cluster and destroy the infection structure of pathogens that invade plant cells. However, $P$. infestans can inhibit the formation of stromules by secreting the effector AVR3a and reduce the contact area with chloroplast (Toufexi et al., 2019). Plant viruses can utilize endogenous host transport machinery to facilitate their intracellular movement (Laporte et al., 2003; Genovés et al., 2010). The AFs play an important role in the formation of stromules, which may be involved in the cell-to-cell transport of the virus in the host (Bhattacharyya and Chakraborty, 2018).

\section{COMMUNICATION BETWEEN CHLORPLAST AND CELL NUCLEUS}

The chloroplasts are important environmental sensors and signaling hubs that transmit the developmental and environmental signals to the nucleus, thus regulating the expression of thousands of nucleus-encoded genes, called retrograde signaling (Chan et al., 2016). The SAL1-PAP retrograde signaling pathway mutant sal1 shows more severe symptoms after infection with either the hemibiotrophic pathogen P. syringae pv. tomato DC3000 or the necrotrophic pathogen Pectobacterium carotovorum subsp. carotovorum EC1, and the ETI responses and the signaling pathways-mediated by SA and JA are attenuated (Ishiga et al., 2017). The metabolite of the plastidial retrograde signaling pathway, methylerythritol cyclodiphosphate (MEcPP), can increase the abundance of the red light receptor phytochrome B protein, then reduce the level and distribution of auxin as well as the content of ethylene, to adjust plant growth. MEcPP mediates the coordination of light and hormone signaling cascades, reprogramming plant growth in response to light (Jiang et al., 2020). The stress-responsive GCN2 kinase phosphorylates the translation initiation factor eIF $2 \alpha$ to control cytosolic messenger RNA (mRNA) translation globally, while the ROS produced by chloroplasts can rapidly activate this process to regulate plant growth under stresses (Lokdarshi et al., 2020). ${ }^{1} \mathrm{O}_{2}$, one of ROS produced by plant cells, can not only cause photodamage of the photosynthetic apparatus but also is related to the retrograde signaling pathways (Asada, 2006; Dogra et al., 2018). The ${ }^{1} \mathrm{O}_{2}$-mediated oxidative posttranslational modification of the ${ }^{1} \mathrm{O}_{2}$ sensor EXECUTER1 (EX1) is necessary to activate the ${ }^{1} \mathrm{O}_{2}$-triggered retrograde signaling pathway (Dogra et al., 2019). The metalloprotease $\mathrm{FtsH} 2$ coordinates the retrograde signaling pathway triggered by ${ }^{1} \mathrm{O}_{2}$ through the proteolysis of EX1 protein (Wang et al., 2016; Dogra et al., 2017).

\section{CHLOROPLAST ARE TARGETED BY PATHOGENS}

Some microbes secrete proteins, named effectors, into plant cells, and certain effectors could enter chloroplasts and target the chloroplast-localized proteins (summarized in Table 1) owing to the leading sequences similar to the host chloroplast transit peptide. Pst_12806 secreted by Puccinia striiformis f. sp. tritici (Xu et al., 2019) interacts with the wheat TaISP protein in chloroplasts (Zheng et al., 2013). ISP protein is a subunit of cytochrome b6-f complex and has a C-terminal Rieske domain, which is responsible for the electron transfer (Yamori et al., 2011). Pst_12806 may weaken the electron transfer ability of the Rieske domain by interacting with the C-terminal of TaISP protein, then inhibits photosynthesis and the by-products ROS production, thereby inhibiting the host cell death and promoting pathogen survival (Xu et al., 2019). Chloroplast-targeted protein 1 (CTP1) secreted by Melampsora larici-populina (Petre et al., 2016) and the secreted protein PvRXLR8620 of Plasmopara viticola 
TABLE 1 | Microbial proteins target plant chloroplasts.

\begin{tabular}{|c|c|c|c|c|}
\hline Microbes & Protein name & Annotation & Implication of interaction & References \\
\hline Alfalfa mosaic virus & $\mathrm{CP}$ & Coat protein & Segregate the chloroplast protein PsbP & Balasubramaniam et al., 2014 \\
\hline Sclerotinia sclerotiorum & ITL & Integrin-like protein & Binding CAS and inhibiting SA synthesis & Tang et al., 2020 \\
\hline Puccinia striiformis & Pst_12806 & Haustorium-specific protein & Interacts with TalSP & Xu et al., 2019 \\
\hline Phytophthora infestans & AVRvnt1 & RxLR type of effector protein & Interacts with and destabilizes GLYK & Gao et al., 2020 \\
\hline Pseudomonas syringae & Hopl1 & Previously named HopPmal & $\begin{array}{l}\text { Causes chloroplast thylakoid structure remodeling } \\
\text { and suppresses SA accumulation }\end{array}$ & Jelenska et al., 2007 \\
\hline Pseudomonas syringae & HopN1 & Cysteine protease & Degraded PsbQ and inhibited PSII activity & Rodríguez-Herva et al., 2012 \\
\hline potato leafroll virus & $\mathrm{CP}$ & Capsid readthrough domain & $\begin{array}{l}\text { Form an extensive interaction network with } \\
\text { chloroplast-localized proteins }\end{array}$ & Deblasio et al., 2018 \\
\hline Tomato yellow leaf curl virus & C4 & & $\begin{array}{l}\text { Inhibit the intercellular spread of RNAi through } \\
\text { interact with BAM1 }\end{array}$ & Rosas-Diaz et al., 2018 \\
\hline Bamboo mosaic virus & Rep & Replicase & Interacts with $\mathrm{NbcpHsp70-2}$ & Huang Y. W. et al., 2017 \\
\hline Pseudomonas syringae & HopZ1a & $\begin{array}{l}\text { HopZ effector family of } \\
\text { cysteine-proteases }\end{array}$ & Suppresses SA and EDS1-dependent resistance. & Macho et al., 2010 \\
\hline Pseudomonas syringae & HopBB1 & Type III effector & Activates TCP14-repressed JA response genes & Yang et al., 2017 \\
\hline Pseudomonas syringae & HopX1 & Cysteine protease & $\begin{array}{l}\text { Interacts with and promotes the degradation of JAZ } \\
\text { proteins }\end{array}$ & Gimenez-lbanez et al., 2014 \\
\hline Ralstonia solanacearum & RipAL & $\begin{array}{l}\text { Contains a putative lipase } \\
\text { domain }\end{array}$ & $\begin{array}{l}\text { Induces jasmonic acid production to suppress } \\
\text { salicylic acid-mediated defense responses }\end{array}$ & Nakano and Mukaihara, 2018 \\
\hline Candidatus Liberibacter asiaticus & SahA & Salicylic acid hydroxylase & Degrade SA and suppress plant defenses & Li et al., 2017 \\
\hline Tomato yellow leaf curl virus & C4 & C4 Protein & Suppression of SA responses & Medina-Puche et al., 2020 \\
\hline Phytophthora infestans & AVR3a & Host-translocated effector & Perturb pathogen induced stromule development & Toufexi et al., 2019 \\
\hline betasatellite & $\beta C 1$ & A suppressor of gene silencing & $\begin{array}{l}\text { Destroy chloroplast ultrastructure and interact with } \\
\text { PsbP to hampers non-specific binding of PsbP to } \\
\text { the geminivirus DNA }\end{array}$ & $\begin{array}{l}\text { Bhattacharyya et al., 2015; } \\
\text { Gnanasekaran et al., } 2019\end{array}$ \\
\hline
\end{tabular}

(Liu et al., 2018) can accumulate in the chloroplasts. Nuclearencoded chloroplast protein glycerate 3-kinase (GLYK) is a basal immunity-related kinase in plants, while the plant pathogen $P$. infestans attempts to interfere with its function by secreting the effector AVRvnt1 (Pais et al., 2018). AVRvnt1 interacts with GLYK and promotes its degradation by the proteasome to prevent it from translocating to the chloroplast. However, the plant NLR protein Rpi-vnt1.1 monitors the transport of GLYK, recognizes AVRvnt1, and triggers immune responses. The lightdependent alternative promoter selection (APS) causes the plant to produce a truncated GLYK under dark conditions, which cannot interact with AVRvnt1 (Gao et al., 2020). SsITL, an integrin-like protein, is secreted by fungal pathogen Sclerotinia sclerotiorum, which suppresses host immune response at the early stage of infection by binding to the Arabidopsis CAS in the chloroplast and inhibiting the accumulation of SA (Zhu et al., 2013; Tang et al., 2020). The early defense response activated by PAMP causes a rapid decrease in non-photochemical quenching (NPQ) (Göhre et al., 2012). However, the oxalic acid secreted by $S$. sclerotiorum acidifies the infected tissue of $A$. thaliana during infection, which limits the protons flux into the stroma, downregulates the activity of ATP synthase, protonizes the PsbS protein, and activates the violaxanthin de-epoxidase (VDE) enzyme to increase NPQ. In addition, violaxanthin, as the precursor of the xanthophyll cycle, plays an important role in the synthesis of the plant hormone ABA (Pasqualini et al., 1999). The activated VDE enzyme catalyzes the conversion from violaxanthin to zeaxanthin, limiting the biosynthesis of $\mathrm{ABA}$, and ultimately affects the plant defense responses, including the production of ROS and callose deposition (Zhou et al., 2015). Rhizoctonia solani causes host chloroplast deformities, weakens the photosynthesis, and perturbs plant hormone signaling to promote the infection (Ghosh et al., 2017). The endophytes in Lolium perenne systemically but moderately increases the hormones biosynthesis and weakens the photosynthesis to increase its resilience (Schmid et al., 2016).

Candidatus Liberibacter asiaticus (CLas) promotes the production of $\mathrm{H}_{2} \mathrm{O}_{2}$ by targeting host chloroplast and inhibits the expression of host antioxidant enzyme genes influencing the detoxification of the host. In addition, through upregulating the expression of ATP synthase, CLas induces the production of ATP in plant cells and then steals ATP to feed itself (Pitino et al., 2017). CLas secretes effector Las5315 (mature protein) to target the chloroplast of the host. In tobacco, it induces cell death and strong callose deposition, which is the main cause for the phloem plugging (Pitino et al., 2016). Moreover, CLas injects an SA hydroxylase SahA to suppress plant defense responses by degrading plant endogenous SA and inhibiting the expression of PR genes, which is similar to the observations in many other bacteria that encode the SA hydroxylases as well (Li et al., 2017). Some SA analogs, due to the structural difference, cannot be degraded by SA hydroxylase; therefore, they can neutralize the effect and effectively control the disease (Palmer et al., 2019). P. syringae delivers effectors into the host cell through the type III secretion system (T3SS) and disrupts the PSII by reprogramming the nuclear-encoded chloroplast-targeted 
genes (NECGs) (De Torres Zabala et al., 2015). HopK1, secreted by $P$. syringae, is a well-studied example that localizes in the chloroplast and can suppress the hypersensitive response ( $\mathrm{Li}$ et al., 2014). Another P. syringae effector HopI1, entering the chloroplasts through a non-canonical pathway, although containing a chloroplast transit peptide sequence, can remodel chloroplast thylakoid structure and inhibit SA-mediated defenses (Jelenska et al., 2007). In addition, HopI1 interacts with the host heat shock protein Hsp70 through its C-terminal J domain, promoting pathogenesis by affecting Hsp70 activity (Jelenska et al., 2010). The cysteine protease HopN1, secreted by $P$. syringae, also locates in the chloroplasts and inhibits host immunity by degrading PsbQ protein and inhibiting PSII activity in tomato (Rodríguez-Herva et al., 2012). Some other effectors, for example, HopZ1a, HopBB1, and HopX1, secreted by $P$. syringae, can activate JA signaling pathway through targeting the JA repressor JAZ (Macho et al., 2009, 2010; Jiang et al., 2013; Gimenez-Ibanez et al., 2014; Yang et al., 2017). Ralstonia solanacearum secretes effector RipAL, which contains a putative lipase domain and targets the chloroplast lipids in plant cell, to promote JA biosynthesis by catalyzing the release of linoleic acid, an important precursor for JA biosynthesis, and succeeds in infection (Nakano and Mukaihara, 2018).

The geminivirus betasatellite that causes radish curl leaf disease (RaLCB) affects the ultrastructure and function of the host chloroplast by secreting $\beta \mathrm{C} 1$ protein to promote disease (Bhattacharyya et al., 2015). Tobacco PsbP protein can bind to geminivirus DNA and activate the defense response against

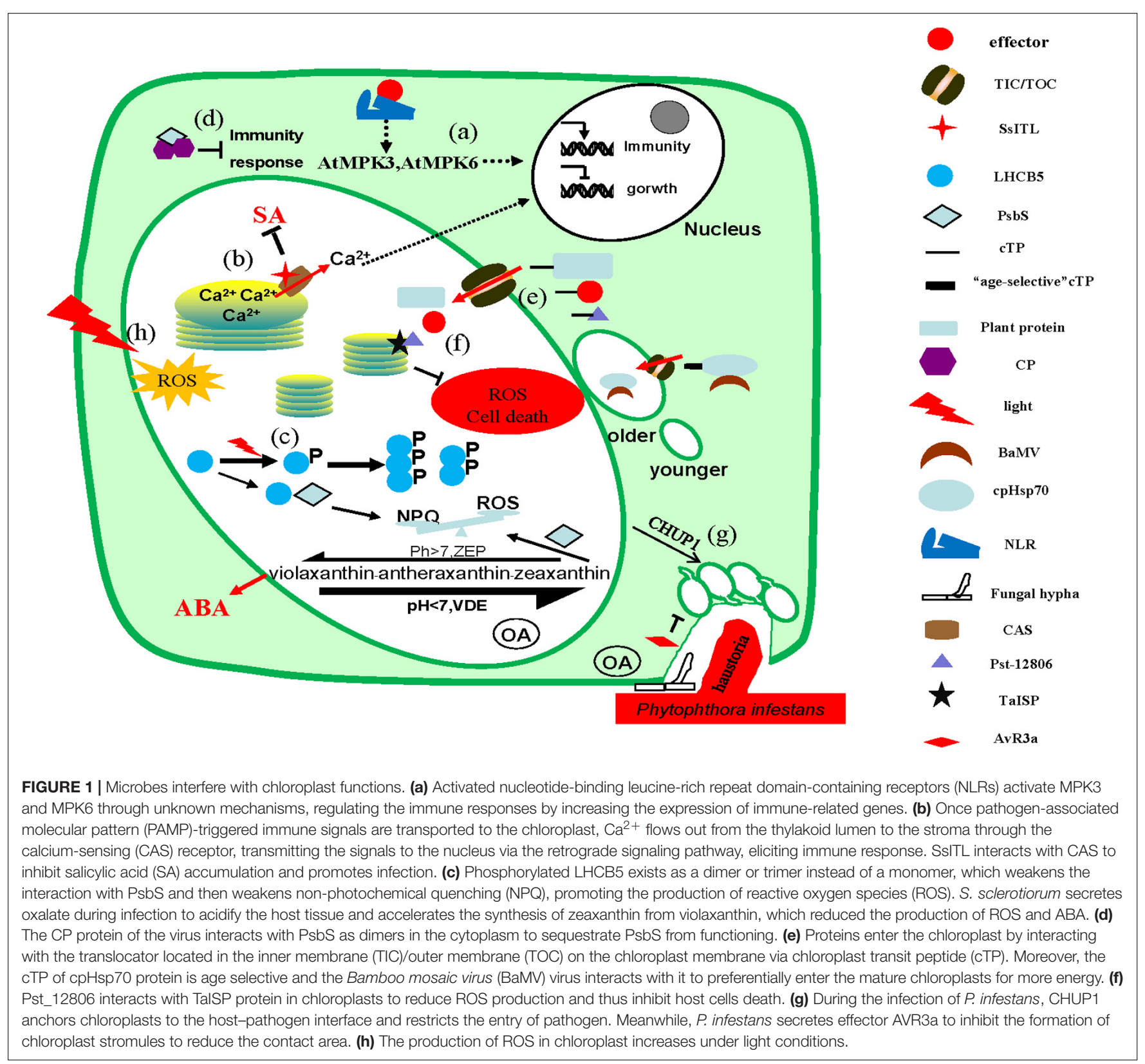


the virus, while $\beta C 1$ interacts with PsbP to prevent its nonspecific binding to geminivirus DNA (Gnanasekaran et al., 2019). The Alfalfa mosaic virus (AMV) coat protein (CP) interacts with the Arabidopsis chloroplast-targeted PsbP protein in the cytoplasm, and overexpression of PsbP greatly reduces the virus accumulation in the infected leaves, suggesting that AMV may use this method to sequestrate PsbP protein to control the induced host resistance (Balasubramaniam et al., 2014). Viruses can promote their replication and movement through target host components (Maule et al., 2002; Pallas and García, 2011; Gray et al., 2014). The chloroplast-localized RNA helicase increased size exclusion limit 2 (ISE2) can regulate the formation and distribution of plasmodesmata. Virus infection can induce the expression of ISE2 in the host. In addition, ISE2 also affects the interaction between the nematode and the host (Ganusova et al., 2017). Potato leafroll virus (PLRV), through the capsid readthrough domain (RTD), establishes extensive interaction networks with host chloroplast-localized proteins to increase its accumulation in host plants (Deblasio et al., 2018). RNAi, as the major antiviral mechanism of plants, can move between cells (Himber, 2003). BARELY ANY MERISTEM 1 (BAM1) and its closest homolog BAM2 in Arabidopsis play an important and redundant role in this process, and the $\mathrm{C} 4$ protein from tomato yellow leaf curl virus (TYLCV) interacts with BAM1 to inhibit the cell-to-cell spread of RNAi (RosasDiaz et al., 2018). Bamboo mosaic virus (BaMV) could regulate the relocalization of NbcpHsp70-2 in N. benthamiana to the chloroplasts in mature stage to enable its infection in mature tobacco leaves (Huang Y. W. et al., 2017). The sensitivity of tobacco to tobacco mosaic virus (TMV) depends on the leaf ages. TMV replicase can interact with the host phloemrelated transcription factors in an age-dependent manner to regulate the transcriptional response to enhance plant phloem loading and the systemic spread of TMV in mature tissues (Collum et al., 2016). The TYLCV encoding a C4 protein relocalizes from the $\mathrm{PM}$ to the chloroplast upon the plant defense activation, interfering with the biosynthesis of SA. This pattern is observed in plant and several plant pathogens from different kingdoms. In plants, there may be a pathway that connects the PM to the chloroplast to activate the defense that is utilized by different pathogens to promote their infection by inhibiting the biosynthesis of SA and possible retrograde signaling (Medina-Puche et al., 2020).

\section{REFERENCES}

Abdelkefi, H., Sugliani, M., Ke, H., Harchouni, S., Soubigou-Taconnat, L., Citerne, S., et al. (2018). Guanosine tetraphosphate modulates salicylic acid signalling and the resistance of arabidopsis thaliana to turnip mosaic virus. Mol. Plant Pathol. 19, 634-646. doi: 10.1111/mpp.12548

Asada, K. (2006). Production and scavenging of reactive oxygen species in chloroplasts and their functions. Plant Physiol. 141, 391-396. doi: 10.1104/pp. 106.082040

Balasubramaniam, M., Kim, B.-S., Hutchens-Williams, H. M., and Loesch-Fries, L. S. (2014). The photosystem ii oxygen-evolving complex protein psbp interacts with the coat protein of alfalfa mosaic virus and inhibits virus replication. Mol. Plant Microbe Interact. 27, 1107-1118. doi: 10.1094/mpmi-0214-0035-r

\section{CONCLUDING REMARKS}

The energy generated by chloroplasts through photosynthesis, including reactions like light absorption, electron transfer, photophosphorylation, and carbon assimilation, not only supports plant growth and development but also participates in plant defense responses. Besides, chloroplasts are also involved in plant immunity through the production of ROS, defense-related hormones, and retrograde signaling pathways. At the same time, chloroplasts are important signaling hubs that communicate with different cell compartments (Figure 1). Because of their important roles, chloroplasts are regarded as obstacles by various "enemies" of plants and is becoming an emerging battlefield in plant-pathogen interactions. Pathogenic microbes interfere with chloroplasts function and structure by delivering small molecules and effector proteins into the plant cells (Table 1). Correspondingly, plants actively exert the defense responses by recognizing the effectors and counteracting the functions of effectors. In the process of chloroplast and host-microbe interaction study, more interesting and fascinating functions of chloroplasts are slowly being elucidated, which has important implications for both plant growth and immunity.

\section{AUTHOR CONTRIBUTIONS}

FY wrote most part of this manuscript. KX helped to writing this manuscript. JL and HP supervised, revised, and complemented the writing.

\section{FUNDING}

This work was supported by the National Key Research and Development Program of China (2019YFE0114200) and the National Natural Science Foundation of China (31972978 and 31772108).

\section{ACKNOWLEDGMENTS}

We thank Drs. Baolei Jia and Gang Yu for critical reading of the manuscript.

Beale, S. I. (2011). Chloroplast signaling: retrograde regulation revelations. Curr. Biol. 21, R391-R393. doi: 10.1016/j.cub.2011.03.072

Beck, C. F. (2005). Signaling pathways from the chloroplast to the nucleus. Planta 222, 743-756. doi: 10.1007/s00425-005-0021-2

Bedard, J. (2005). Recognition and envelope translocation of chloroplast preproteins. J. Exp. Bot. 56, 2287-2320. doi: 10.1093/jxb/eri243

Bender, C. L., Alarco'n-Chaidez, F., and Gross, D. C. (1999). Pseudomonas syringae phytotoxins: mode of action, regulation, and biosynthesis by peptide and polyketide synthetases. Microbiol. Mol. Biol. Rev. 63, 266-292.

Bender, C. L., Palmer, D. A., Peñaloza-Vázquez, A., Rangaswamy, V., and Ullrich, M. (1998). Biosynthesis and regulation of coronatine, a non-host-specific phytotoxin produced by pseudomonas syringae. Subcell. Biochem. 29, 321-341. doi: 10.1007/978-1-4899-1707-2_10 
Bhattacharyya, D., and Chakraborty, S. (2018). Chloroplast: the trojan horse in plant-virus interaction. Mol. Plant Pathol. 19, 504-518. doi: 10.1111/mpp. 12533

Bhattacharyya, D., Gnanasekaran, P., Kumar, R. K., Kushwaha, N. K., Sharma, V. K., Yusuf, M. A., et al. (2015). A geminivirus betasatellite damages the structural and functional integrity of chloroplasts leading to symptom formation and inhibition of photosynthesis. J. Exp. Bot. 66, 5881-5895. doi: 10.1093/jxb/erv299

Bruce, B. D. (2000). Chloroplast transit peptides: structure, function and evolution. Trends Cell Biol. 10, 440-447. doi: 10.1016/s0962-8924(00)01833-x

Caplan, J. L., Kumar, A. S., Park, E., Padmanabhan, M. S., Hoban, K., Modla, S., et al. (2015). Chloroplast stromules function during innate immunity. Dev. Cell 34, 45-57. doi: 10.1016/j.devcel.2015.05.011

Caplan, J. L., Mamillapalli, P., Burch-Smith, T. M., Czymmek, K., and DineshKumar, S. P. (2008). Chloroplastic protein nripl mediates innate immune receptor recognition of a viral effector. Cell 132, 449-462. doi: 10.1016/j.cell. 2007.12.031

Cavalier-Smith, T. (2002). Chloroplast evolution: secondary symbiogenesis and multiple losses. Curr. Biol. 12, R62-R64. doi: 10.1016/s0960-9822(01)00675-3

Chan, K. X., Phua, S. Y., Crisp, P., McQuinn, R., and Pogson, B. J. (2016). Learning the languages of the chloroplast: retrograde signaling and beyond. Annu. Rev. Plant Biol. 67, 25-53.

Chen, Y.-C., Holmes, E. C., Rajniak, J., Kim, J.-G., Tang, S., Fischer, C. R., et al. (2018). N-hydroxy-pipecolic acid is a mobile metabolite that induces systemic disease resistance in arabidopsis. Proc. Natl. Acad. Sci. U.S.A. 115, E4920-E4929. doi: 10.1073/pnas.1805291115

Collum, T. D., Padmanabhan, M. S., Hsieh, Y.-C., and Culver, J. N. (2016). Tobacco mosaic virus-directed reprogramming of auxin/indole acetic acid protein transcriptional responses enhances virus phloem loading. Proc. Natl. Acad. Sci. U.S.A. 113, E2740-E2749. doi: 10.1073/pnas.1524390113

Daniell, H., Lin, C.-S., Yu, M., and Chang, W.-J. (2016). Chloroplast genomes: diversity, evolution, and applications in genetic engineering. Genome Biol. 17:134. doi: 10.1186/s13059-016-1004-2

Day, P. M., and Theg, S. M. (2018). Evolution of protein transport to the chloroplast envelope membranes. Photosynth. Res. 138, 315-326. doi: 10.1007/s11120-0180540-x

De Torres Zabala, M., Littlejohn, G., Jayaraman, S., Studholme, D., Bailey, T., Lawson, T., et al. (2015). Chloroplasts play a central role in plant defence and are targeted by pathogen effectors. Nat. Plants 1:15074. doi: 10.1038/nplants. 2015.74

Deblasio, S. L., Rebelo, A. R., Parks, K., Gray, S. M., and Heck, M. C. (2018). Disruption of chloroplast function through downregulation of phytoene desaturase enhances the systemic accumulation of an aphid-borne, phloemrestricted virus. Mol. Plant Microbe Interact. 31, 1095-1110. doi: 10.1094/ mpmi-03-18-0057-r

Dempsey, D. M. A., Vlot, A. C., Wildermuth, M. C., and Klessig, D. F. (2011). Salicylic acid biosynthesis and metabolism. Arabidopsis Book 9:e0156. doi: 10. $1199 /$ tab.0156

Ding, X., Jimenez-Gongora, T., Krenz, B., and Lozano-Duran, R. (2019). Chloroplast clustering around the nucleus is a general response to pathogen perception in nicotiana benthamiana. Mol. Plant Pathol. 20, 1298-1306. doi: $10.1111 / \mathrm{mpp} .12840$

Dogra, V., Duan, J., Lee, K. P., Lv, S., Liu, R., and Kim, C. (2017). Ftsh2-dependent proteolysis of executerl is essential in mediating singlet oxygen-triggered retrograde signaling in arabidopsis thaliana. Front. Plant Sci. 8:1145. doi: 10. 3389/fpls.2017.01145

Dogra, V., Li, M., Singh, S., Li, M., and Kim, C. (2019). Oxidative post-translational modification of executer 1 is required for singlet oxygen sensing in plastids. Nat. Commun. 10:2834. doi: 10.1038/s41467-019-10760-6

Dogra, V., Rochaix, J.-D., and Kim, C. (2018). Singlet oxygen-triggered chloroplastto-nucleus retrograde signalling pathways: an emerging perspective. Plant Cell Environ. 41, 1727-1738. doi: 10.1111/pce.13332

Gaffney, T., Friedrich, L., Vernooij, B., Negrotto, D., Nye, G., Uknes, S., et al. (1993). Requirement of salicylic acid for the induction of systemic acquired resistance. Science 261, 754-756. doi: 10.1126/science.261.5122.754

Ganusova, E., Rice, J. H., Carlew, T. S., Patel, A., Perrodin-Njoku, E., Hewezi, T., et al. (2017). Altered expression of a chloroplast protein affects the outcome of virus and nematode infection. Mol. Plant Microbe Interact. 30, 478-488. doi: 10.1094/mpmi-02-17-0031-r

Gao, C., Xu, H., Huang, J., Sun, B., Zhang, F., Savage, Z., et al. (2020). Pathogen manipulation of chloroplast function triggers a light-dependent immune recognition. Proc. Natl. Acad. Sci. U.S.A. 117, 9613-9620. doi: 10.1073/pnas. 2002759117

Genovés, A., Navarro, J. A., and Pallás, V. (2010). The intra- and intercellular movement of melon necrotic spot virus (mnsv) depends on an active secretory pathway. Mol. Plant Microbe Interact. 23, 263-272. doi: 10.1094/mpmi-23-30263

Ghosh, S., Kanwar, P., and Jha, G. (2017). Alterations in rice chloroplast integrity, photosynthesis and metabolome associated with pathogenesis of Rhizoctonia solani. Sci. Rep. 7:41610. doi: 10.1038/srep41610

Gimenez-Ibanez, S., Boter, M., Fernández-Barbero, G., Chini, A., Rathjen, J. P., and Solano, R. (2014). The bacterial effector hopxl targets jaz transcriptional repressors to activate jasmonate signaling and promote infection in arabidopsis. PLoS Biol. 12:e1001792. doi: 10.1371/journal.pbio.1001792

Glazebrook, J. (2005). Contrasting mechanisms of defense against biotrophic and necrotrophic pathogens. Annu. Rev. Phytopathol. 43, 205-227. doi: 10.1146/ annurev.phyto.43.040204.135923

Gnanasekaran, P., Ponnusamy, K., and Chakraborty, S. (2019). A geminivirus betasatellite encoded betacl protein interacts with psbp and subverts psbpmediated antiviral defence in plants. Mol. Plant Pathol. 20, 943-960. doi: 10. 1111/mpp.12804

Göhre, V., Jones, A. M. E., Sklenáø, J., Robatzek, S., and Weber, A. P. M. (2012). Molecular crosstalk between PAMP-triggered immunity and photosynthesis. Mol. Plant Microbe Interact. 25, 1083-1092.

Govrin, E. M., and Levine, A. (2000). The hypersensitive response facilitates plant infection by the necrotrophic pathogen botrytis cinerea. Curr. Biol. 10, 751-757. doi: 10.1016/s0960-9822(00)00560-1

Gray, M. W. (1989). The evolutionary origins of organdies. Trends Genet. 5, 294-299.

Gray, S., Cilia, M., and Ghanim, M. (2014). Circulative, "nonpropagative” virus transmission: an orchestra of virus-, insect-, and plant-derived instruments. Adv. Virus Res. 89, 141-199. doi: 10.1016/b978-0-12-800172-1.00004-5

Hanson, M. R., and Hines, K. M. (2018). Stromules: probing formation and function. Plant Physiol. 176, 128-137. doi: 10.1104/pp.17.01287

Himber, C. (2003). Transitivity-dependent and -independent cell-to-cell movement of rna silencing. EMBO J. 22, 4523-4533. doi: 10.1093/emboj/ $\operatorname{cdg} 431$

Huang, H., Liu, B., Liu, L., and Song, S. (2017). Jasmonate action in plant growth and development. J. Exp. Bot. 68, 1349-1359. doi: 10.1093/jxb/erw495

Huang, Y. W., Hu, C. C., Tsai, C. H., Lin, N. S., and Hsu, Y. H. (2017). Chloroplast hsp70 isoform is required for age-dependent tissue preference of bamboo mosaic virus in mature nicotiana benthamiana leaves. Mol. Plant Microbe Interact. 30, 631-645. doi: 10.1094/mpmi-01-17-0012-r

Ishiga, Y., Watanabe, M., Ishiga, T., Tohge, T., Matsuura, T., Ikeda, Y., et al. (2017). The sal-pap chloroplast retrograde pathway contributes to plant immunity by regulating glucosinolate pathway and phytohormone signaling. Mol. Plant Microbe Interact. 30, 829-841. doi: 10.1094/MPMI-03-17-0055-R

Jarvis, P., and Robinson, C. (2004). Mechanisms of protein import and routing in chloroplasts. Curr. Biol. 14, R1064-R1077. doi: 10.1016/j.cub.2004.11.049

Jarvis, P., and Soll, J. (2001). Toc, tic, and chloroplast protein import. Biochim. Biophys. Acta. 1541, 64-79. doi: 10.1016/s0167-4889(01)00147-1

Jelenska, J., van Hal, J. A., and Greenberg, J. T. (2010). Pseudomonas syringae hijacks plant stress chaperone machinery for virulence. Proc. Natl. Acad. Sci. U.S.A. 107, 13177-13182. doi: 10.1073/pnas.0910943107

Jelenska, J., Yao, N., Vinatzer, B. A., Wright, C. M., Brodsky, J. L., and Greenberg, J. T. (2007). A j domain virulence effector of pseudomonas syringae remodels host chloroplasts and suppresses defenses. Curr. Biol. 17, 499-508. doi: 10.1016/ j.cub.2007.02.028

Jiang, J., Xiao, Y., Chen, H., Hu, W., Zeng, L., Ke, H., et al. (2020). Retrograde induction of phyb orchestrates ethylene-auxin hierarchy to regulate growth. Plant Physiol. 183, 1268-1280. doi: 10.1104/pp.20.00090

Jiang, S., Yao, J., Ma, K.-W., Zhou, H., Song, J., He, S. Y., et al. (2013). Bacterial effector activates jasmonate signaling by directly targeting jaz transcriptional repressors. PLoS Pathog. 9:e1003715. doi: 10.1371/journal.ppat.1003715 
Jones, J. D. G., and Dangl, J. L. (2006). The plant immune system. Nature 444, 323-329. doi: 10.1038/nature05286

Kim, C., and Apel, K. (2013). 1O2-mediated and EXECUTER-dependent retrograde plastid-to-nucleus signaling in norflurazon-treated seedlings of Arabidopsis thaliana. Mol. Plant. 6, 1580-1591. doi: 10.1093/mp/sst020

Kim, Y., Gilmour, S. J., Chao, L., Park, S., and Thomashow, M. F. (2020). Arabidopsis camta transcription factors regulate pipecolic acid biosynthesis and priming of immunity genes. Mol. Plant 13, 157-168. doi: 10.1016/j.molp.2019. 11.001

Krieger-Liszkay, A., Kós, P. B., and Hideg, É (2011). Superoxide anion radicals generated by methylviologen in photosystem i damage photosystem ii. Physiol. Plant. 142, 17-25. doi: 10.1111/j.1399-3054.2010.01416.x

Kumar, A. S., Park, E., Nedo, A., Alqarni, A., Ren, L., Hoban, K., et al. (2018). Stromule extension along microtubules coordinated with actin-mediated anchoring guides perinuclear chloroplast movement during innate immunity. Elife 7:e23625. doi: 10.7554/elife.23625

Kwok, E. Y., and Hanson, M. R. (2004). Plastids and stromules interact with the nucleus and cell membrane in vascular plants. Plant Cell Rep. 23, 188-195. doi: 10.1007/s00299-004-0824-9

Laporte, C., Vetter, G., Loudes, A.-M., Robinson, D. G., Hillmer, S., Stussi-Garaud, C., et al. (2003). Involvement of the secretory pathway and the cytoskeleton in intracellular targeting and tubule assembly of grapevine fanleaf virus movement protein in tobacco by-2 cells. Plant Cell 15, 2058-2075. doi: 10.1105/tpc.013896

Li, G., Froehlich, J. E., Elowsky, C., Msanne, J., Ostosh, A. C., Zhang, C., et al. (2014). Distinct pseudomonas type-iii effectors use a cleavable transit peptide to target chloroplasts. Plant J. 77, 310-321. doi: 10.1111/tpj.12396

Li, J., Pang, Z., Trivedi, P., Zhou, X., Ying, X., Jia, H., et al. (2017). 'Candidatus liberibacter asiaticus' encodes a functional salicylic acid (sa) hydroxylase that degrades sa to suppress plant defenses. Mol. Plant Microbe Interact. 30, 620-630. doi: 10.1094/MPMI-12-16-0257-R

Li, N., Han, X., Feng, D., Yuan, D., and Huang, L.-J. (2019). Signaling crosstalk between salicylic acid and ethylene/jasmonate in plant defense: do we understand what they are whispering? Int. J. Mol. Sci. 20:671. doi: 10.3390/ ijms20030671

Lim, C., Baek, W., Jung, J., Kim, J.-H., and Lee, S. (2015). Function of aba in stomatal defense against biotic and drought stresses. Int. J. Mol. Sci. 16, 15251-15270. doi: 10.3390/ijms160715251

Liu, C., Cui, D., Zhao, J., Liu, N., Wang, B., Liu, J., et al. (2019). Two Arabidopsis receptor-like cytoplasmic kinases sze1 and sze2 associate with the zar1-zed1 complex and are required for effector-triggered immunity. Mol. Plant 12, 967-983. doi: 10.1016/j.molp.2019.03.012

Liu, M., Zhang, S., Hu, J., Sun, W., Padilla, J., He, Y., et al. (2019). Phosphorylationguarded light-harvesting complex ii contributes to broad-spectrum blast resistance in rice. Proc. Natl. Acad. Sci. U.S.A. 116, 17572-17577. doi: 10.1073/ pnas. 1905123116

Liu, Y., Lan, X., Song, S., Yin, L., Dry, I. B., Qu, J., et al. (2018). In planta functional analysis and subcellular localization of the oomycete pathogen plasmopara viticola candidate rxlr effector repertoire. Front. Plant Sci. 9:286. doi: 10.3389/ fpls.2018.00286

Lokdarshi, A., Guan, J., Urquidi Camacho, R. A., Cho, S. K., Morgan, P. W., Leonard, M., et al. (2020). Light activates the translational regulatory kinase $\mathrm{gcn} 2$ via reactive oxygen species emanating from the chloroplast. Plant Cell 32, 1161-1178. doi: 10.1105/tpc. 19.00751

Macho, A. P., Guevara, C. M., Tornero, P., Ruiz-Albert, J., and Beuzón, C. R. (2010). The pseudomonas syringae effector protein hopzla suppresses effectortriggered immunity. New Phytol. 187, 1018-1033. doi: 10.1111/j.1469-8137. 2010.03381.x

Macho, A. P., Ruiz-Albert, J., Tornero, P., and Beuzón, C. R. (2009). Identification of new type iii effectors and analysis of the plant response by competitive index. Mol. Plant Pathol. 10, 69-80. doi: 10.1111/j.1364-3703.2008.00511.x

Martin, W., Rujan, T., Richly, E., Hansen, A., Cornelsen, S., Lins, T., et al. (2002). Evolutionary analysis of arabidopsis, cyanobacterial, and chloroplast genomes reveals plastid phylogeny and thousands of cyanobacterial genes in the nucleus. Proc. Natl. Acad. Sci. U.S.A. 99, 12246-12251. doi: 10.1073/pnas.182432999

Maule, A., Leh, V., and Lederer, C. (2002). The dialogue between viruses and hosts in compatible interactions. Curr. Opin. Plant Biol. 5, 279-284. doi: 10.1016/ s1369-5266(02)00272-8
McFadden, G. I. (2001). Chloroplast origin and integration. Plant Physiol. 125, 50-53. doi: 10.1104/pp.125.1.50

Medina-Puche, L., Tan, H., Dogra, V., Wu, M., Rosas-Diaz, T., Wang, L., et al. (2020). A defense pathway linking plasma membrane and chloroplasts and co-opted by pathogens. Cell 182, 1109-1124.e25. doi: 10.1016/j.cell.2020.07.020

Nakano, M., and Mukaihara, T. (2018). Ralstonia solanacearum type iii effector ripal targets chloroplasts and induces jasmonic acid production to suppress salicylic acid-mediated defense responses in plants. Plant Cell Physiol. 59, 2576-2589. doi: $10.1093 /$ pcp/pcy177

Natesan, S. K., Sullivan, J. A., and Gray, J. C. (2005). Stromules: a characteristic cell-specific feature of plastid morphology. J. Exp. Bot. 56, 787-797.

Nomura, H., Komori, T., Uemura, S., Kanda, Y., Shimotani, K., Nakai, K., et al. (2012). Chloroplast-mediated activation of plant immune signalling in Arabidopsis. Nat. Commun. 3:926. doi: 10.1038/ncomms1926

Pais, M., Yoshida, K., Giannakopoulou, A., Pel, M. A., Cano, L. M., Oliva, R. F., et al. (2018). Gene expression polymorphism underpins evasion of host immunity in an asexual lineage of the irish potato famine pathogen. BMC Evol. Biol. 18:93. doi: 10.1186/s12862-018-1201-6

Pallas, V., and García, J. A. (2011). How do plant viruses induce disease? Interactions and interference with host components. J. Gen. Virol. 92, 26912705. doi: 10.1099/vir.0.034603-0

Palmer, I. A., Chen, H., Chen, J., Chang, M., Li, M., Liu, F., et al. (2019). Novel salicylic acid analogs induce a potent defense response in arabidopsis. Int. J. Mol. Sci. 20:3356. doi: 10.3390/ijms20133356

Pasqualini, S., Batini, P., Ederli, L., and Antonielli, M. (1999). Responses of the xanthophyll cycle pool and ascorbate-glutathione cycle to ozone stress in two tobacco cultivars. Free Radic. Res. 31(Suppl.), S67-S73. doi: 10.1080/ 10715769900301341

Peng, Y., Van Wersch, R., and Zhang, Y. (2018). Convergent and divergent signaling in pamp-triggered immunity and effector-triggered immunity. Mol. Plant Microbe Interact. 31, 403-409. doi: 10.1094/mpmi-06-17-0145-cr

Petre, B., Lorrain, C., Saunders, D. G. O., Win, J., Sklenar, J., Duplessis, S., et al. (2016). Rust fungal effectors mimic host transit peptides to translocate into chloroplasts. Cell Microbiol. 18, 453-465. doi: 10.1111/cmi.12530

Pierella Karlusich, J. J., Zurbriggen, M. D., Shahinnia, F., Sonnewald, S., Sonnewald, U., Hosseini, S. A., et al. (2017). Chloroplast redox status modulates genomewide plant responses during the non-host interaction of tobacco with the hemibiotrophic bacterium xanthomonas campestris pv. Vesicatoria. Front. Plant Sci. 8:1158. doi: 10.3389/fpls.2017.01158

Pitino, M., Armstrong, C. M., Cano, L. M., and Duan, Y. (2016). Transient expression of candidatus liberibacter asiaticus effector induces cell death in nicotiana benthamiana. Front. Plant Sci. 7:982. doi: 10.3389/fpls.2016.00982

Pitino, M., Armstrong, C. M., and Duan, Y. (2017). Molecular mechanisms behind the accumulation of atp and $\mathrm{h} 2 \mathrm{o} 2$ in citrus plants in response to 'candidatus liberibacter asiaticus' infection. Hortic. Res. 4:17040. doi: 10.1038/hortres. 2017.40

Pospíšil, P., Arató, A., Krieger-Liszkay, A., and Rutherford, A. W. (2004). Hydroxyl radical generation by photosystem ii†. Biochemistry 43, 6783-6792. doi: 10 . 1021/bi036219i

Ranf, S., Eschen-Lippold, L., Pecher, P., Lee, J., and Scheel, D. (2011). Interplay between calcium signalling and early signalling elements during defence responses to microbe- or damage-associated molecular patterns. Plant J. 68, 100-113. doi: 10.1111/j.1365-313x.2011.04671.x

Reinbothe, S., Mache, R., and Reinbothe, C. (2000). A second, substrate-dependent site of protein import into chloroplasts. Proc. Natl. Acad. Sci. U.S.A. 97, 9795-9800. doi: 10.1073/pnas.160242597

Rekhter, D., Lüdke, D., Ding, Y., Feussner, K., Zienkiewicz, K., Lipka, V., et al. (2019). Isochorismate-derived biosynthesis of the plant stress hormone salicylic acid. Science 365, 498-502. doi: 10.1126/science.aaw1720

Richardson, L. G. L., Small, E. L., Inoue, H., and Schnell, D. J. (2018). Molecular topology of the transit peptide during chloroplast protein import. Plant Cell 30, 1789-1806. doi: 10.1105/tpc.18.00172

Richly, E., and Leister, D. (2004). An improved prediction of chloroplast proteins reveals diversities and commonalities in the chloroplast proteomes of arabidopsis and rice. Gene 329, 11-16. doi: 10.1016/j.gene.2004.01.008

Rodríguez-Herva, J. J., González-Melendi, P., Cuartas-Lanza, R., Antúnez-Lamas, M., Río-Alvarez, I., Li, Z., et al. (2012). A bacterial cysteine protease effector 
protein interferes with photosynthesis to suppress plant innate immune responses. Cell. Microbiol. 14, 669-681. doi: 10.1111/j.1462-5822.2012.01749.x

Rosas-Diaz, T., Zhang, D., Fan, P., Wang, L., Ding, X., Jiang, Y., et al. (2018). A virus-targeted plant receptor-like kinase promotes cell-to-cell spread of rnai. Proc. Natl. Acad. Sci. U.S.A. 115, 1388-1393. doi: 10.1073/pnas.1715556115

Rossi, F. R., Krapp, A. R., Bisaro, F., Maiale, S. J., Pieckenstain, F. L., and Carrillo, N. (2017). Reactive oxygen species generated in chloroplasts contribute to tobacco leaf infection by the necrotrophic fungus botrytis cinerea. Plant J. 92, 761-773. doi: $10.1111 /$ tpj. 13718

Schmid, J., Day, R., Zhang, N., Dupont, P.-Y., Cox, M. P., Schardl, C. L., et al. (2016). Host tissue environment directs activities of an epichloë endophyte, while it induces systemic hormone and defense responses in its native perennial ryegrass host. Mol. Plant Microbe Interact. 30, 138-149. doi: 10.1094/mpmi-1016-0215-r

Seo, M., and Koshiba, T. (2002). Complex regulation of ABA biosynthesis in plants. Trends Plant Sci. 7, 41-48. doi: 10.1016/s1360-1385(01)02187-2

Serrano, I., Audran, C., and Rivas, S. (2016). Chloroplasts at work during plant innate immunity. J. Exp. Bot. 67, 3845-3854. doi: 10.1093/jxb/erw088

Seybold, H., Trempel, F., Ranf, S., Scheel, D., Romeis, T., and Lee, J. (2014). $\mathrm{Ca} 2+$ signalling in plant immune response: from pattern recognition receptors to ca2+decoding mechanisms. New Phytol. 204, 782-790. doi: 10.1111/nph. 13031

Sheng, X., Liu, X., Cao, P., Li, M., and Liu, Z. (2018). Structural roles of lipid molecules in the assembly of plant psii-lhcii supercomplex. Biophys. Rep. 4, 189-203. doi: 10.1007/s41048-018-0068-9

Singh, R., Lee, S., Ortega, L., Ramu, V. S., Senthil-Kumar, M., Blancaflor, E. B., et al. (2018). Two chloroplast-localized proteins: atnhr2a and atnhr2b, contribute to callose deposition during nonhost disease resistance in Arabidopsis. Mol. Plant Microbe Interact. 31, 1280-1290. doi: 10.1094/mpmi-04-18-0094- r

Su, J., Yang, L., Zhu, Q., Wu, H., He, Y., Liu, Y., et al. (2018). Active photosynthetic inhibition mediated by mpk3/mpk6 is critical to effector-triggered immunity. PLoS Biol. 16:e2004122. doi: 10.1371/journal.pbio.2004122

Sun, L., Ren, H., Liu, R., Li, B., Wu, T., Sun, F., et al. (2010). An h-type thioredoxin functions in tobacco defense responses to two species of viruses and an abiotic oxidative stress. Mol. Plant Microbe Interact. 23, 1470-1485. doi: 10.1094/ mpmi-01-10-0029

Sun, T., Huang, J., Xu, Y., Verma, V., Jing, B., Sun, Y., et al. (2020). Redundant CAMTA transcription factors negatively regulate the biosynthesis of salicylic acid and n-hydroxypipecolic acid by modulating the expression of sard 1 and cbp60g. Mol. Plant 13, 144-156. doi: 10.1016/j.molp.2019.10.016

Tang, L., Yang, G., Ma, M., Liu, X., Li, B., Xie, J., et al. (2020). An effector of a necrotrophic fungal pathogen targets the calcium-sensing receptor in chloroplasts to inhibit host resistance. Mol. Plant Pathol. 21, 686-701. doi: 10.1111/mpp. 12922

Teng, Y. S., Chan, P. T., and Li, H. M. (2012). Differential age-dependent import regulation by signal peptides. PLoS Biol. 10:e1001416. doi: 10.1371/journal.pbio. 1001416

Théry, C., Ostrowski, M., and Segura, E. (2009). Membrane vesicles as conveyors of immune responses. Nat. Rev. Immunol. 9, 581-593. doi: 10.1038/nri2567

Tian, W., Hou, C., Ren, Z., Wang, C., Zhao, F., Dahlbeck, D., et al. (2019). A calmodulin-gated calcium channel links pathogen patterns to plant immunity. Nature 572, 131-135. doi: 10.1038/s41586-019-1413-y

Torrens-Spence, M. P., Bobokalonova, A., Carballo, V., Glinkerman, C. M., Pluskal, T., Shen, A., et al. (2019). Pbs3 and eps1 complete salicylic acid biosynthesis from isochorismate in Arabidopsis. Mol. Plant 12, 1577-1586. doi: 10.1016/j. molp.2019.11.005

Toufexi, A., Duggan, C., Pandey, P., Savage, Z., Segretin, M. E., Yuen, L. H., et al. (2019). Chloroplasts navigate towards the pathogen interface 1 to counteract infection by the Irish potato famine pathogen. bioRxiv [Preprint] doi: 10.1101/ 516443

Wang, J., Liu, X., Zhang, A., Ren, Y., Wu, F., Wang, G., et al. (2019). A cyclic nucleotide-gated channel mediates cytoplasmic calcium elevation and disease resistance in rice. Cell Res. 29, 820-831. doi: 10.1038/s41422-019-0219-7

Wang, L., Kim, C., Xu, X., Piskurewicz, U., Dogra, V., Singh, S., et al. (2016). Singlet oxygen- and executer 1-mediated signaling is initiated in grana margins and depends on the protease ftsh2. Proc. Natl. Acad. Sci. U.S.A. 113:E3792. doi: $10.1073 /$ pnas. 1603562113

Wang, M., Rui, L., Yan, H., Shi, H., Zhao, W., Lin, J. E., et al. (2018). The major leaf ferredoxin $\mathrm{fd} 2$ regulates plant innate immunity in Arabidopsis. Mol. Plant Pathol. 19, 1377-1390. doi: 10.1111/mpp.12621

Wasternack, C., and Song, S. (2017). Jasmonates: biosynthesis, metabolism, and signaling by proteins activating and repressing transciption. J. Exp. Bot. 68, 1303-1321. doi: 10.1093/jxb/erw443

Xu, Q., Tang, C., Wang, X., Sun, S., Zhao, J., Kang, Z., et al. (2019). An effector protein of the wheat stripe rust fungus targets chloroplasts and suppresses chloroplast function. Nat. Commun. 10:5571. doi: 10.1038/s41467-019-13 487-6

Xu, Y. H., Liu, R., Yan, L., Liu, Z. Q., Jiang, S. C., Shen, Y. Y., et al. (2012). Lightharvesting chlorophyll a/b-binding proteins are required for stomatal response to abscisic acid in Arabidopsis. J. Exp. Bot. 63, 1095-1106. doi: 10.1093/jxb/ err315

Yamori, W., Takahashi, S., Makino, A., Price, G. D., Badger, M. R., and Von Caemmerer, S. (2011). The roles of atp synthase and the cytochrome b6/f complexes in limiting chloroplast electron transport and determining photosynthetic capacity. Plant Physiol. 155, 956-962. doi: 10.1104/pp.110. 168435

Yang, L., Teixeira, P. J. P. L., Biswas, S., Finkel, O. M., He, Y., Salas-Gonzalez, I., et al. (2017). Pseudomonas syringae type iii effector hopbb1 promotes host transcriptional repressor degradation to regulate phytohormone responses and virulence. Cell Host Microbe 21, 156-168. doi: 10.1016/j.chom.2017.01.003

Zheng, W., Huang, L., Huang, J., Wang, X., Chen, X., Zhao, J., et al. (2013). High genome heterozygosity and endemic genetic recombination in the wheat stripe rust fungus. Nat. Commun. 4:2673. doi: 10.1038/ncomms3673

Zhou, J., Zeng, L., Liu, J., and Xing, D. (2015). Manipulation of the xanthophyll cycle increases plant susceptibility to sclerotinia sclerotiorum. PLoS Pathog. 11:e1004878. doi: 10.1371/journal.ppat.1004878

Zhu, W., Wei, W., Fu, Y., Cheng, J., Xie, J., Li, G., et al. (2013). A secretory protein of necrotrophic fungus sclerotinia sclerotiorum that suppresses host resistance. PLoS One 8:e53901. doi: 10.1371/journal.pone.005 3901

Ziehe, D., Dünschede, B., and Schünemann, D. (2017). From bacteria to chloroplasts: evolution of the chloroplast srp system. Biol. Chem. 398, 653-661. doi: 10.1515/hsz-2016-0292

Zipfel, C. (2014). Plant pattern-recognition receptors. Trends Immunol. 35, 345351. doi: 10.1016/j.it.2014.05.004

Conflict of Interest: The authors declare that the research was conducted in the absence of any commercial or financial relationships that could be construed as a potential conflict of interest.

Copyright (C) 2021 Yang, Xiao, Pan and Liu. This is an open-access article distributed under the terms of the Creative Commons Attribution License (CC BY). The use, distribution or reproduction in other forums is permitted, provided the original author(s) and the copyright owner(s) are credited and that the original publication in this journal is cited, in accordance with accepted academic practice. No use, distribution or reproduction is permitted which does not comply with these terms. 